\title{
Clima laboral y satisfacción laboral en los docentes de una universidad pública y una privada
}

\author{
Mg. Ana Alegre Morales \\ Mg. Rocío Barrientos Montellanos \\ Mg. Marilú Milla Rotta
}

La presente investigación tuvo el objetivo de analizar la relación entre el clima y la satisfacción laboral en docentes de una universidad pública y una privada. La muestra fue de 62 docentes de la Universidad Federico Villarreal y 38 docentes de la Universidad Champagnat, a quienes se les aplicó el Cuestionario de Clima Laboral (CLA) y el Cuestionario de Satisfacción Laboral versión S20/23. Se halló que los docentes de institución privada tienen mejor clima laboral que los de institución pública $(p=.000)$ y también presentan mayor satisfacción laboral $(p=.000)$. Además, los resultados indicaron que existen una relación significativa entre las variables de estudio solamente en la institución privada $(p=.003)$.

Palabras clave: Clima laboral, Docentes universitarios, Satisfacción laboral, Universidad pública, Universidad privada.

The objective of this study was to analyze the relationship between working environment and job satisfaction in teaching at a public university and one private. The sample was 62 teachers at the University Federico Villarreal and 38 teachers of the University Champagnat, who were assessment with the Working Environment Questionnaire and Job Satisfaction Questionnaire S20/23. It waS found that teachers in private institutions have better working environment than public institution $(p=.000)$ and also have higher job satisfaction $(p=.000)$. In addition, the results indicated that there is a significant relationship between both variables only in private institution $(p=.003)$.

Keywords: Job satisfaction, University teachers, Public university, Private university, Working environment. 


\section{INTRODUCCIÓN}

El concepto, la utilidad y función del trabajo han sido objeto de grandes controversias a lo largo de la historia. En la mayor parte de las sociedades, la generalidad de las personas debe adecuarse a las exigencias del trabajo tales como la capacitación, actualización, especialización, dominio de idiomas y tecnología acordes con los avances de la ciencia, así como desempeñar algún tipo de trabajo y en algún tipo de empresa u organización.

Al mismo tiempo, este quehacer es considerado como una actividad de vastos alcances que se refleja e influye sobre casi todos los aspectos de la conducta humana, por lo que los individuos al desempeñarlo no solo trasladan a él sus habilidades intelectuales y motrices, sino también sus individualidades y sus relaciones con el entorno y los demás colaboradores.

Por otro lado, el trabajo brinda al individuo la sensación de pertenencia a un sector de la sociedad laboral que se considera importante, necesario y valioso. Es en este contexto, que destaca la importancia del clima laboral, que se percibe en la organización a la que se pertenece, en la que los diferentes aspectos que la integran y la relación del mismo pueden convertirse en una fuerza impulsora y un elemento de vital importancia y el trabajo no se convierta en una actividad estresante y opresora.

Además, la satisfacción frente al trabajo trae diversas consecuencias positivas, destacando entre ellas un mejor desempeño que le compete tanto a él como a la empresa y tomarlo en cuenta como uno más de los factores necesarios para lograr una producción mayor, la cual sería un beneficio y cuyos frutos se dirigen principalmente a la empresa; pero también al trabajador como parte de su realización profesional y personal. 


\section{MARCO TEÓRICO DE REFERENCIA}

\section{Definición de clima laboral}

Hoy en día, en el campo laboral es importante considerar aspectos que permitan que el trabajo dentro de las organizaciones se dé de la manera más adecuada y que al interior de ellas las relaciones entre los trabajadores en su diaria convivencia sean en la medida de lo posible, buenas o agradables y que, por consiguiente, contribuyan y den como resultado final la consecución de los objetivos trazados y los resultados esperados.

Al revisar las investigaciones hechas por Caligiore y Díaz, 2003; Gómez, 2004; Chiang, Salazar y Núñez, 2007; Arangú, 2008 y Monteza (2012) se refieren al clima laboral utilizando los términos de clima laboral y clima organizacional, como el trato que el empleador puede tener con sus colaboradores, como la relación entre los miembros del personal de la organización y/o de la institución.

Con respecto al clima en la organización, Chiavenato (2000) señala que:

El clima organizacional se refiere al ambiente interno existente entre los miembros de la organización, está estrechamente ligado al grado de motivación de los empleados e indica de manera específica las propiedades motivacionales del ambiente organizacional. (Chiavenato, 2000, p.126)

Es así que el clima aparece desde hace unas décadas como uno de los elementos fundamentales para mejorar las relaciones entre los colaboradores y la organización entendiéndolo como la atmósfera que reina en el ambiente laboral, el entorno en el que se desarrolla el trabajo, el fruto de la relación de la empresa con sus trabajadores.

El clima laboral se ve afectado por muchas variables según lo manifiesta Henríquez (2002), quien señaló que:

El clima influye en la motivación, el desempeño y la satisfacción en el empleo. Esto lo hace creando ciertas clases de expectativas con respecto a qué consecuencias se generan a través de diferentes acciones. Los empleados en la organización esperan ciertas recompensas, satisfacciones y frustraciones basándose en la percepción que tiene el clima en la organización". (Henríquez, 2002, p.29)

Es así como, por su relación con la satisfacción laboral y por ende con la productividad y el desempeño, su análisis parte principalmente de los diferentes elementos que lo integran, por la revisión de las teorías y dimensiones del mismo.

Martínez (2001) señala que "El clima laboral está determinado por el conjunto de factores vinculados a la calidad de vida dentro de una organización. Constituye una percepción, y como tal adquiere valor de realidad en las organizaciones" (p. 32). En ese sentido, Soto (2001) manifiesta que "La percepción es el proceso activo de percibir la realidad y organizarla en interpretaciones o visiones sensatas" (p.11). Es la forma en que un empleado percibe el ambiente que lo rodea, se refiere a 
las características del medio ambiente de trabajo, las que son percibidas, directa o indirectamente por los trabajadores y causan repercusiones en el comportamiento laboral.

Por lo tanto, se concluye que la variable a utilizar en esta investigación es clima laboral y se puede inferir que el concepto de percepción es un término importante; ya que el clima laboral está determinado por las percepciones que tienen los trabajadores con respecto a la organización, dicho de otra manera, son las interpretaciones que los colaboradores se forman de la organización a la que pertenecen.

El clima laboral es producto de los diferentes aspectos objetivos tales como la estructura, así como los procesos organizacionales y los aspectos subjetivos en que destacan las conductas y las experiencias de los trabajadores. Esta naturaleza multidimensionalidad del clima hace que las variables no sean siempre fáciles de definir, debido a la interacción que se produce entre ellas, lo que dificulta el proceso de aislamiento conceptual de la misma (Ramos, Peiró y Ripoll, 1996).

A medida que se han ido construyendo instrumentos para su medición es que los autores han ido determinando cierta cantidad de dimensiones o características que influyen en el comportamiento de los individuos y afectan el ambiente de las organizaciones.

El instrumento que se utilizó en la investigación fue el Cuestionario de Clima Laboral (CLA) elaborado por los consultores del grupo TEA-CEGOS con el objetivo de disponer de un instrumento acorde con las tendencias modernas en materia de gestión de los recursos humanos adaptado a las características laborales en función de la experiencia consultora. Este cuestionario se basa en la teoría de Blake y Mounton $(1981,1994)$, distingue dos ejes principales en la gestión empresarial y en las formas de dirigir, una está orientado hacia la persona y es la que recoge aquellas dimensiones del clima laboral que tienen más relación con las actitudes y vivencias personales, refiriéndose más al grado de satisfacción del trabajador y menos a su inserción en la organización ; el otro eje está orientado a la empresa u organización y reúne las dimensiones del clima laboral que dependen en mayor medida del funcionamiento y organización de la entidad, afectan a la satisfacción 0 insatisfacción de los empleados y, por tanto, al clima laboral, dependiendo principalmente de la forma en que la institución está organizada y dirigida (Corral y Pereña, 2003).

Los análisis estadísticos realizados han permitido confirmar empíricamente la existencia de estos dos ejes. El desarrollo del CLA ha sido aplicado en diversas empresas y contextos lo que ha permitido contar con datos significativos y diversos para estudiar las características psicométricas del instrumento.

\section{Definición de satisfacción laboral}

Uno de los aspectos importantes que deben tomarse en cuenta en el desempeño de una profesión y más en el ámbito educativo es el de conocer cuan satisfecho se está con la labor que se realiza. En ese contexto, la satisfacción laboral docente puede encontrarse como un indicador y elemento para el mejoramiento de la institución, como organización y el docente como un colaborador eficiente si se concibe que la satisfacción pueda ser un factor importante en la vinculación y compromiso del mismo con la organización y el trabajo en ella. 
Por su parte, Crites (citado por Rodríguez et al., 2009) la definió como el estado afectivo en el sentido de gusto o disgusto general, que la persona muestra hacia su trabajo.

Locke (citado por Ovejero, 2006) señaló a la satisfacción como un estado placentero y positivo, resultante de la valoración del trabajo o de las experiencias laborales del sujeto.

Allí radicaría la importancia de conocer los factores que implican y la posibilidad de establecer una relación mayor con otros aspectos del trabajo. En ese sentido, Morán (2005) en relación a la definición dada por Locke supone la aceptación de que la satisfacción es un constructo global, que abarca facetas específicas de satisfacción tales como el trabajo en sí, salario e incentivos, sistema de supervisión, las oportunidades de promoción, las condiciones ambientales de trabajo, etc.

Finalmente, la satisfacción laboral es una respuesta emocional o afectiva en la que inciden una serie de factores, sean actitudes, experiencias con una carga emocional determinante para el sujeto. Por ello, la importancia de conocer el nivel de satisfacción laboral y más aún qué factores pueden causar satisfacción o insatisfacción, resultan importantes en nuestro estudio.

Entre las teorías que explican la satisfacción laboral se encuentran:

La teoría bifactorial o Teoría de los dos factores (Motivación - Higiene). Esta teoría fue desarrollada por Herzberg, Mausner, y Snyderman (1959), quienes demostraron que ciertos factores estaban asociados con la satisfacción y otros, con la insatisfacción. La primera compuesta por factores motivacionales tales como el reconocimiento, ser creativo en el trabajo, tener responsabilidades respecto a los demás y la promoción; mientras que los que son responsables de la insatisfacción se catalogan como factores de higiene: política de la organización, aspectos técnicos de la supervisión, relaciones interpersonales, salario y condiciones de trabajo. Los primeros intrínsecos al trabajo, mientras los segundos son extrínsecos, así lo señala (Rodríguez et al., 2009).

En el contexto educativo esta teoría ha permitido determinar los factores que influyen en la satisfacción o no del docente y en función de él lograr mayor participación y productividad, tomando como elementos al liderazgo, las relaciones grupales y los incentivos.

La teoría de Lawler que se basa en la teoría de la equidad de Adams. Considera que la satisfacción en el trabajo depende de la diferencia existente entre lo que la persona considera justo y equitativo recibir como consecuencia de su trabajo y lo que esa misma persona considera que efectivamente recibe (en términos de paga, responsabilidad, promoción entre otros) como consecuencia de su trabajo (Nadler y Lawler, 1977).

Por otra parte, en cuanto a la medición de la satisfacción laboral, durante años se han ido construyendo diferentes instrumentos como el Cuestionario General de Satisfacción en Organizaciones Laborales conocido como \$4/82 que incluía aspectos específicos culturales y organizacionales, así como el obtener un índice global y específicos para diversas facetas de la satisfacción laboral con una aplicación muy amplia, que al considerar 82 ítems y ser muy amplio se logró reducirlo en la versión S20/23 que consta de 23 ítems con la misma utilidad diagnóstica, fiabilidad y validez que el anterior. Dicho cuestionario establece cinco dimensiones para la satisfacción considerando (Meliá y Peiró, 1998): 
- Satisfacción con la supervisión, referente a la forma en que los superiores juzgan la tarea, la supervisión recibida, la proximidad y frecuencia de la supervisión, el apoyo recibido de los superiores, las relaciones personales con los mismos y a la igualdad y justicia de trato recibida de la empresa.

- Satisfacción con el ambiente físico, referente al espacio en el lugar de trabajo, limpieza, higiene, salubridad, temperatura, ventilación, iluminación, entre otros.

- Satisfacción con las prestaciones, referente al grado en que la empresa cumple el convenio, la forma en que se da la negociación, el salario recibido, las oportunidades de promoción y las de formación.

- Satisfacción intrínseca, referente al trabajo por sí mismo, las oportunidades que ofrece el trabajo de hacer aquello que gusta o en lo que se destaca, los objetivos, metas y producción a alcanzar.

- Satisfacción con la participación en las decisiones del grupo de trabajo, del departamento o sección o de la propia tarea.

\section{METODOLOGÍA}

La presente investigación es de tipo transversal y de un solo corte muestral. El diseño es descriptivo transversal correlacional bivariable. Se aplicó un cuestionario para medir cada una de las variables, recoger la información de los participantes y establecer las relaciones entre las variables. Además, el diseño es comparativo porque describe las diferencias en cuanto al clima laboral y la satisfacción laboral considerando el tipo de gestión.

El estudio se realizó en la ciudad de Lima con docentes del nivel superior procedentes de la universidad pública Federico Villarreal y la universidad privada Marcelino Champagnat.

Se aplicó dos instrumentos que midieron por un lado el clima laboral y por el otro, la satisfacción laboral, lo que nos permitió lograr resultados muy importantes para la investigación.

\section{RESULTADOS}

La muestra es de 62 docentes de la institución pública: Universidad Federico Villarreal y de 38 docentes de la institución privada: Universidad Champagnat.

Se presentan e interpretan los resultados obtenidos luego de realizado el análisis estadístico. 
Gráfico 1 Nivel de clima laboral para las instituciones públicas y privadas

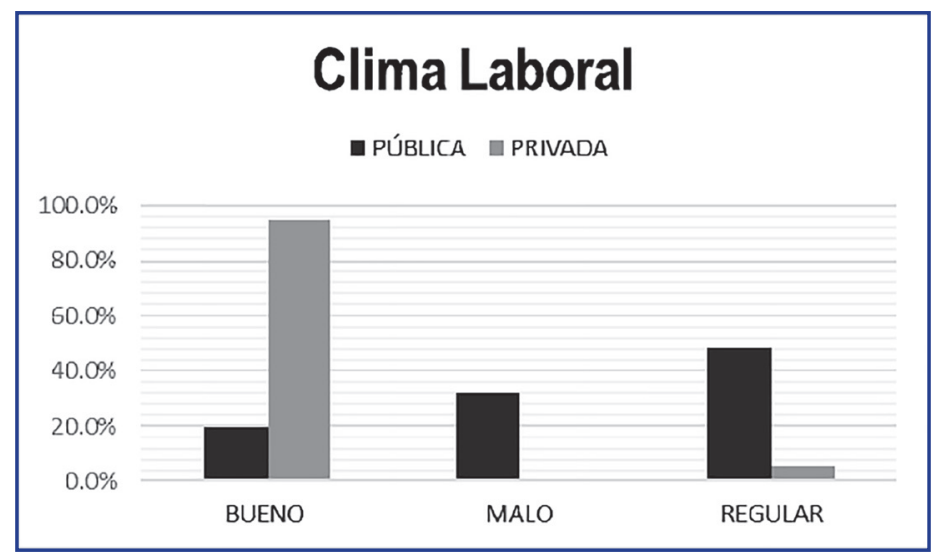

Se observa en el gráfico 1, que el nivel de clima laboral de los docentes en la institución privada es bueno en un $94.7 \%$ y regular en un 5.3\%; mientras que en la institución pública el $48.4 \%$ de los docentes tiene un regular nivel de clima laboral, el $32.3 \%$ malo y el $19.4 \%$ bueno.

Gráfico 2 Nivel en las dimensiones de clima laboral para las instituciones públicas y privadas

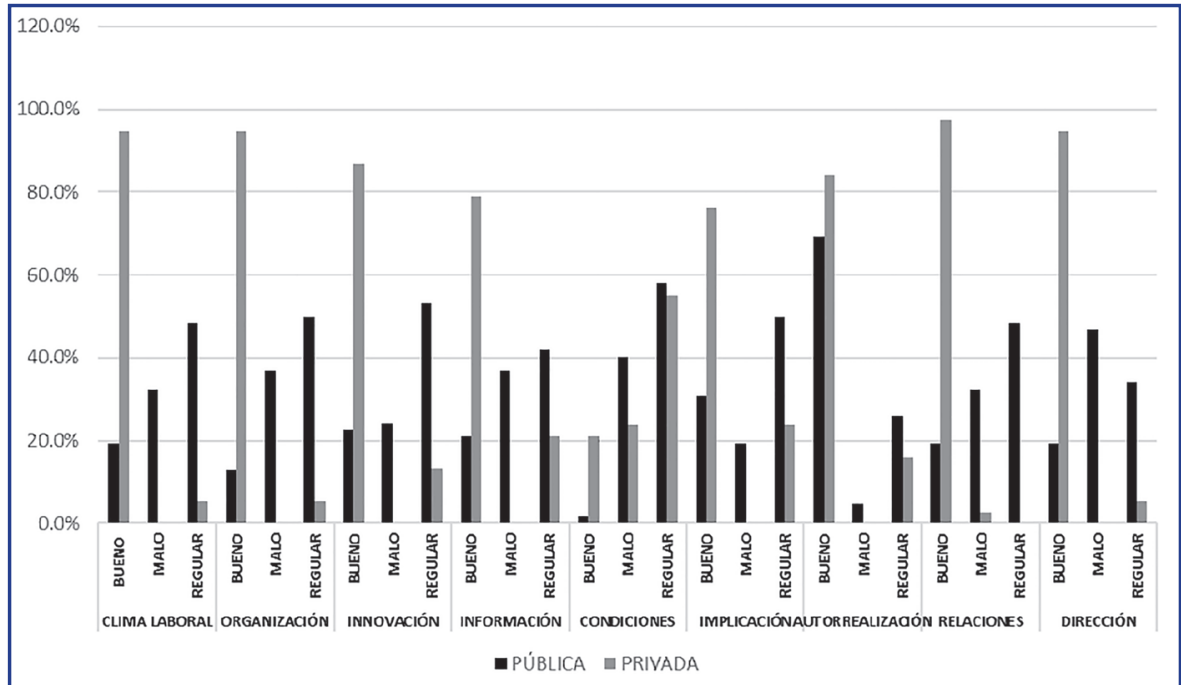

Las diferencias que existen en clima laboral de las instituciones públicas y privadas se puede observar en el gráfico 2 en el que se muestran los resultados obtenidos en cada una de sus dimensiones. 
Gráfico 3 Nivel de satisfacción laboral para las instituciones públicas y privadas

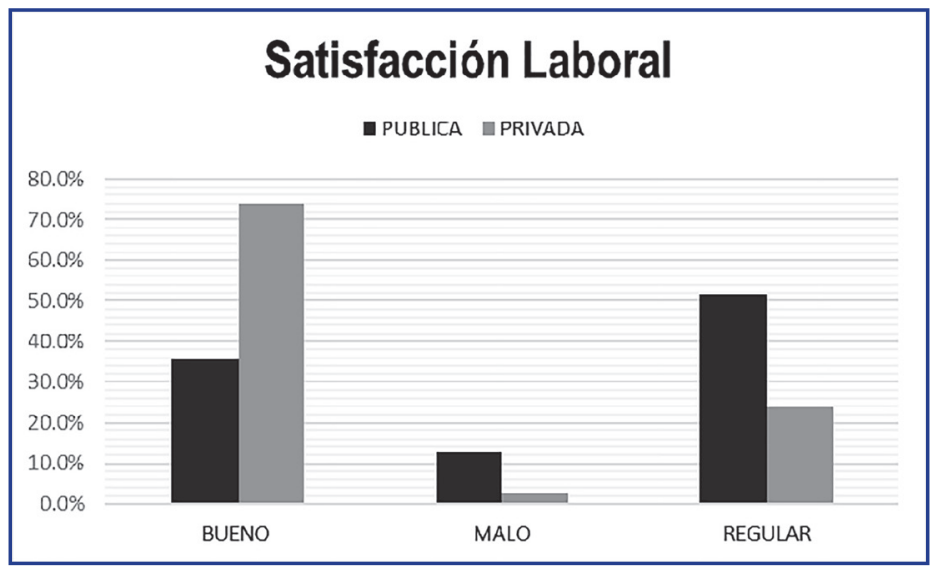

Se observa en el gráfico 3, que el nivel de satisfacción laboral de los docentes en la institución privada es bueno en un $73.7 \%, 23.7$ es regular y 2.6 es malo; mientras que en la institución pública el $51.6 \%$ de los docentes tiene un regular nivel de clima laboral, el $12.9 \%$ malo y el $35.5 \%$ bueno.

Gráfico 4 Nivel en las dimensiones de satisfacción laboral para las instituciones públicas y privadas

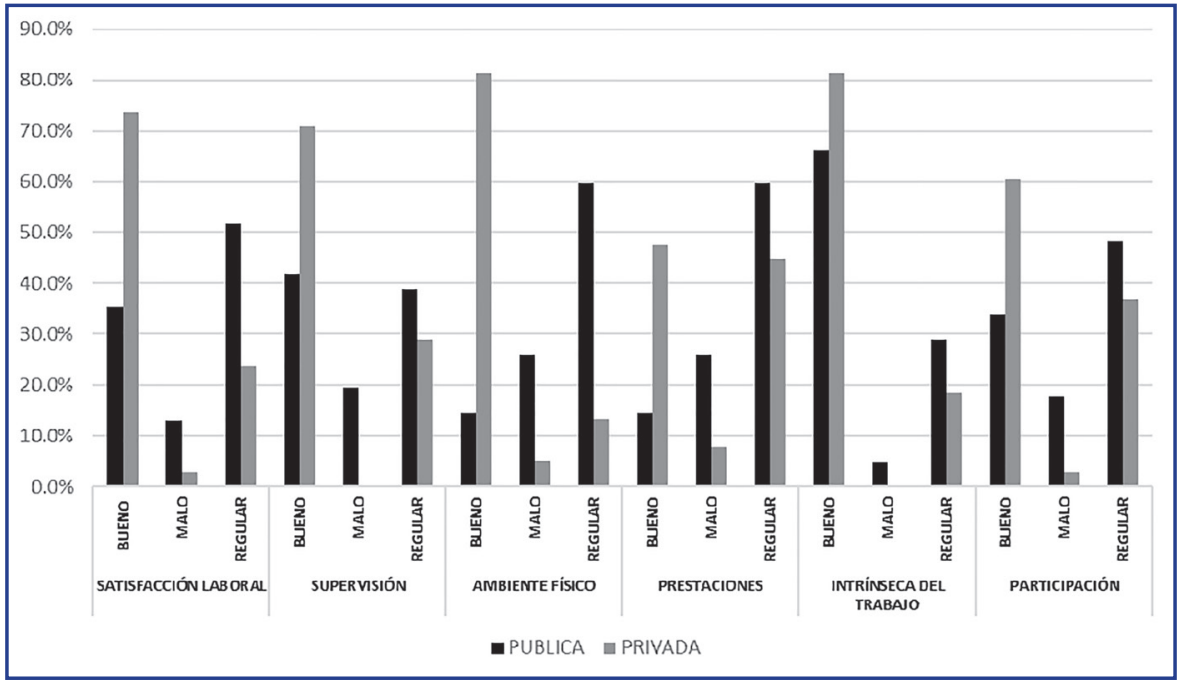

Las diferencias que existen en satisfacción laboral de las instituciones públicas y privadas se puede observar en el gráfico 4 en el que se muestran los resultados obtenidos en cada una de sus dimensiones. 


\section{DISCUSIÓN}

El análisis de datos apoya que los docentes de las instituciones privadas consideran su clima laboral como bueno mientras que los docentes de las instituciones públicas consideran que la institución le ofrece un clima laboral regular. Es relevante también mencionar que un tercio de los docentes de las instituciones públicas califican que dicha institución brinda un clima laboral malo. Este hallazgo podría explicarse debido a que en las universidades públicas existiría un funcionamiento organizacional inadecuado lo cual no estaría favoreciendo su eficiencia, asimismo, su estilo gerencial rígido, la falta de supervisión y los problemas interpersonales generarían un clima tenso y poco productivo (Caligiore y Díaz, 2003).

Del mismo modo, estos resultados se explicarían por las características gerenciales, tomando las modalidades de gestión de las empresas, en las instituciones privadas. En este marco, la institución educativa privada está preocupada en mejorar sus niveles de clima organizacional, identificando y reforzando la percepción de los trabajadores con respecto a esta variable y formulando acciones para su mejora (Arangú, 2008).

Cabe destacar que en las instituciones privadas existe una mayor preocupación por el recurso humano, ya que consideran que ello influye en la calidad y optimización de los servicios que brindan. Existe una cultura en la que se promueven relaciones armónicas entre las personas, la gerencia y los trabajadores, creando así un buen clima laboral (Pérez, 2010).

El análisis de datos muestra un mayor nivel de satisfacción laboral en los docentes de las instituciones privadas (que la califica de buena) con respecto al nivel de percepción de las instituciones públicas (que la califica de regular).

Al respecto, este resultado se explicaría debido a que en las instituciones privadas existen mejores condiciones físicas para trabajo, en comparación a las instituciones públicas; además, de las remuneraciones, promociones y/o ascensos (Fernández y Paravic, 2003), lo cual se confirma con los hallazgos de Silva (2009) quien reportó que dos tercios del personal docente de una universidad pública mostraban insatisfacción en su centro laboral y sensación de frustración.

Del mismo modo, otro factor que explicaría las diferencias entre docentes de institución pública y privada sería la remuneración que es superior en las instituciones privadas. Al respecto, Canal (2013) demostró que a medida que aumentaban los ingresos, aumentaba la satisfacción. Del mismo modo, Olivares et al. (2006) determinaron que la satisfacción con la institución era buena; mientras que la satisfacción por la remuneración, por la tensión laboral, por la condición laboral y satisfacción laboral global fueron regulares.

Finalmente, con respecto a la hipótesis general en la que se planteó la existencia de una relación significativa entre el clima laboral y la satisfacción laboral en los docentes de una universidad pública y una privada, fue probada.

La relación general entre ambas variables se encuentra en concordancia con otras investigaciones en las que también se hallaron relaciones significativas entre el clima laboral y la satisfacción laboral (Chiang, Salazar y Núñez, 2007; Silva, 2009; Monteza, 2012). Por otro lado, el hallazgo que indicó 
que esta relación significativa sólo se presentó en los docentes de instituciones privadas y no en los docentes de instituciones públicas, guarda concordancia con las diferencias significativas presentadas entre ambos grupos de docentes, a favor de los que laboran en la institución privada.

Al parecer la insatisfacción con su centro laboral y sensación de frustración que presentan los docentes de instituciones públicas (Silva, 2009), al igual que la falta de retroalimentación de los procesos que determinan los comportamientos organizacionales y la ausencia de cambios planificados para mejorar el clima organizacional (Monteza, 2012) explicarían los hallazgos de la presente investigación.

\section{CONCLUSIONES}

Existe relación significativa entre el clima laboral y la satisfacción laboral; siendo ésta directamente proporcional determinándose que las instituciones privadas presentan una opinión de mayor nivel de clima laboral con un mayor nivel de satisfacción laboral, mientras que en las instituciones públicas solo hay una regular consideración del clima laboral.

Existen diferencias significativas en la percepción del clima laboral, organización, innovación, información, condiciones, implicación, autorrealización, relaciones y dirección de los docentes de una universidad privada y una pública. Siendo las Instituciones privadas las que cuentan con un mayor nivel de organización, de innovación, de información, de implicación, de autorrealización, de relaciones y de dirección con respecto a las públicas.

Existen diferencias significativas en la percepción de la satisfacción laboral, satisfacción con la supervisión, satisfacción con el ambiente físico del trabajo, satisfacción en las prestaciones y satisfacción con la participación de los docentes de una universidad privada y una pública. Además, nos permite establecer que son las instituciones privadas las que cuentan con un mayor nivel de satisfacción laboral, de satisfacción con la supervisión, satisfacción con el ambiente físico, de satisfacción en la prestación de servicios y de satisfacción con la participación con respecto a las públicas.

Existen diferencias significativas entre las dimensiones del clima laboral y las dimensiones de la satisfacción laboral entre organización y las dimensiones de satisfacción con la supervisión, satisfacción con el ambiente físico y satisfacción intrínseca del trabajo en los docentes de una universidad privada y una pública, además las instituciones privadas son las que cuentan con un mayor nivel de entre Información y de satisfacción con la supervisión, satisfacción con el ambiente físico, satisfacción intrínseca del trabajo y satisfacción con la participación.

\section{RECOMENDACIONES}

Se recomienda a las instituciones públicas, establecer políticas que permitan al docente involucrarse y participar en el proceso organizacional de la institución. Las políticas deberán proyectarse por toda la institución a fin de captar al personal para trabajar sobre la importancia del eje organizacional y los climas de trabajos favorables como factores claves de éxito. 
Se recomienda en el marco de la Nueva Ley Universitaria brindar una adecuada acreditación de su funcionamiento organizacional para que mediante los principios y valores de la organización, determinados a través de un diagnóstico o una auditoría de valores y de manera consciente, los docentes realmente asuman el vínculo que llevará a la organización hacia el éxito. En caso contrario, criticar algunas veces los valores de una organización que pueden ser reajustados formalmente, basados en discusiones dirigidas por el grupo representativo de la Asamblea Universitaria de la institución.

Se recomienda diseñar y poner en práctica un verdadero programa de inducción que permita al docente visualizar un escenario global de la institución en la percepción del clima laboral para la organización, innovación, información, condiciones, implicación, autorrealización, relaciones y dirección de los docentes de la universidad, especialmente en la pública.

Se recomienda implementar, en lo posible y de acuerdo a las normas, una política de remuneración y beneficios equitativos, contratación transparente, de promoción incentivadora y de fiscalización objetiva para suprimir toda fuente de frustración y resentimiento por parte del personal, para establecer una adecuada relación entre el clima laboral y la satisfacción de los docentes, en especial en las instituciones públicas.

\section{REFERENCIAS}

Brunet, L. (1999). El clima de trabajo en las organizaciones. México D.F., México: Trillas.

Canal, J. (2013). Ingresos y satisfacción laboral de los trabajadores españoles con título de doctor. En: Revista española de investigaciones sociológicas. -- N. 144, p49-72. 48p.

Chiang, M., Salazar, C., y Núñez, A. (2007). Clima organizacional y satisfacción laboral en instituciones públicas: Adaptación y ampliación de un instrumento. Recuperado de http:// dialnet.unirioja.es/descarga/articulo/2234840.pdf

Gómez., C. A. (2004). Diseño, construcción y validación de un instrumento que evalúa el clima organizacional en empresas colombianas, desde la respuesta al ítem (Tesis de grado, Universidad Católica de Colombia, Bogotá, Colombia).

Gutiérrez Martínez F: Motivaciones, desempeño y satisfacción laboral del potencial humano en la Universidad Peruana Los Andes. http://www.planificación.upla.edu.pe/portal/images/ DOC.\%20PDF/motivación.pdf 
Hernández, R., Fernández, C. y Baptista, P. (1999). Metodología de la investigación. (2a. ed.).México, McGraw-Hill Interamericana Editores, S.A. de C.V.

Kinicki, A. y Kreitner, R. (2003). Comportamiento organizacional: Conceptos, problemas y prácticas. México D.F., México: McGraw-Hill Interamericana.

Kreinert, R. y Kinicki, A. (1995). Comportamiento de las organizaciones. (Primera edición española de la tercera edición en inglés). Madrid, España: Clamades.

Martínez, L. (2001). Análisis del clima laboral: una herramienta de Gestión imprescindible. (4ta ed.). [En línea] Disponible en: http://www.monografias.com/trabajos6/medicli/medicli.shtml.

Meliá, J. L. y Peiró, J. M. (1998).Cuestionario de satisfacción laboral S4/82.Valencia, España: Universidad de Valencia.

Morán, C. (2005). Psicología del trabajo. Madrid, España: Ediciones Aljibe.

Pritchard, R. y Karasick, B. (1973). The effect of organizational climate on managerial job performance and job satisfaction. Organizational Behavior and Human Performance. En R. Ponce (Ed.). Fundamentos de derecho (2a ed.). México D.F., México: Banca y Comercio.

Ramos, J., Peiró, J., y Ripoll, P. (1996). Condiciones de trabajo y clima laboral, en J. Peiró y F. Prieto (eds.), Tratado de Psicología del Trabajo, Vol. 1: La actividad laboral en su contexto (pp. 38-91). Madrid, España: Síntesis.

Rodríguez, A., Zarco, M. V., y Gonzáles J. M. (2009). Psicología del trabajo. Madrid, España: Ediciones Pirámide.

Sánchez J. M.: (2005) Influencia de la gestión universitaria en el clima organizacional: un estudio comparativo de tres universidades. [Tesis Grado de Magister en Administración con mención en gestión empresarial]. Facultad de Ciencias Administrativas. Universidad Nacional Mayor de San Marcos; 2005. 